\title{
ZEROS OF SUCCESSIVE DERIVATIVES AND ITERATED OPERATORS ON ANALYTIC FUNCTIONS
}

\author{
J. K. SHAW AND C. L. PRATHER
}

\begin{abstract}
For a function $f$ analytic in the closed disc $|z|<1$, we study the behavior of zeros of the successive iterates $\left(\theta^{n} f\right)(z), n=0,1,2, \ldots$, where $\theta=$ $(z+\alpha)^{p+1} d / d z$. We find that such behavior closely parallels that for the ordinary derivative operator. Using change-of-variable methods, we obtain information on zeros of derivatives of functions analytic in half-planes.
\end{abstract}

1. Introduction. Let $f$ be analytic in the unit disc $|z|<1$. A well-known principle in function theory is that $f$ cannot have too many derivatives vanishing too near $z=0$, unless $f$ is a polynomial. The study of this phenomenon is the theory which has been associated with the names of Gončarov and Whittaker [2]-[5]. Its principal feature is the existence of zero-free neighborhoods of $z=0$. There is an absolute constant $G$, known as the Goncarov constant, such that if $f$ is analytic in $|z|<1$, is not a polynomial, and $\varepsilon>0$, then there is an infinite sequence of derivatives $f^{\left(n_{k}\right)}(z)$ which do not vanish in the discs $|z|<(G-\varepsilon) /\left(n_{k}+1\right)$. The exact value of $G$ is unknown, but it is known to lie between .7259 and .7378 .

In the present paper, we consider the analogous problem for the case of the differential operator $\theta=(z+\alpha)^{p+1} d / d z$, where $p>0$ and where $\alpha$ denotes a complex number. Taking $|\alpha|<1$ and $f(z)$ analytic in a neighborhood of the closed unit disc $|z|<1$, we study the zero-free regions of the iterates $\left(\theta^{n} f\right)(z), n=$ $0,1,2, \ldots$ The neighborhoods of $z=-\alpha$ are the most interesting, for in this case all but a finite number (not just an infinite number) of the iterates $\left(\theta^{n} f\right)(z)$ are nonzero in punctured discs which shrink with increasing $n$ to the point $z=-\alpha$.

The results we obtain for differentiation do not arise from taking $p=-1$ in the definition of $\theta$. Instead, we use other values of $p$ and employ change-of-variable methods to get information about zeros of derivatives of functions analytic in regions of the plane other than discs. Such problems have been studied by Widder [7, Theorem 31 and corollary, pp. 166-167] for functions analytic at $\infty$, and for functions analytic in half-planes and representable as Laplace Transforms, and a simpler proof of a result implied by Widder has recently been given by Boas [1]. This result can be stated as follows.

TheOREM A ([1], [7]). Let $F(w)=\sum_{n=1}^{\infty} b_{n} w^{-n}$ be analytic at $\infty$, with $F$ nonconstant. Then there is a constant $c>0$ such that for all $n$ sufficiently large, $F^{(n)}(w)$ has no finite zero outside the circle $|w|=n c$.

Received by the editors May 30, 1979 and, in revised form, July 18, 1979.

AMS (MOS) subject classifications (1970). Primary 30A08; Secondary 30A86.

Key words and phrases. Zeros of derivatives, zero-free regions, differential operators.

(c) 1980 American Mathematical Society 0002-9939/80/0000-0265/\$03.00 
As $F$ is analytic in $|z|>R$ in Theorem $\mathrm{A}$, the constant $c$ depends on $F$ and $R$.

Our results yield this theorem as a special case. We also obtain Widder's description of the radial distribution of zeros [7, Theorem 35], and we get additional theorems on periodic functions, where Widder's method does not apply. These applications are given in $\$ 3$.

2. The $\theta$-operator. We shall obtain an integral representation for the $\boldsymbol{n}$ th iterate $\left(\theta^{n} f\right)(z)$. There are various ways of getting to the end result; for example, one could extend the method used by Hille [6, Vol. 2, p. 51] for the operator $z d / d z$. We will use an alternate approach.

We do this by expressing $\theta^{n}\left[z^{k}\right]$ as a function of $(z+\alpha)^{1+n p}$ having polynomial coefficients, as given in (2.2), and then express the polynomials as integral transforms. Then the integral representation for $\theta^{n} f$ is obtained by the usual power series method.

For each nonnegative integer $m$, we have $\theta\left[(z+\alpha)^{m}\right]=m(z+\alpha)^{m+p}$, and more generally,

$$
\begin{aligned}
\theta^{n}\left[(z+\alpha)^{m}\right] & =(m)(m+p)(m+2 p) \cdots(m+(n-1) p)(z+\alpha)^{m+n p} \\
& =C_{m n}^{(p)}(z+\alpha)^{m+n p}, \quad m>0, n \geqslant 1,
\end{aligned}
$$

where $C_{m n}^{(p)}=(m)(m+p)(m+2 p) \cdots(m+(n-1) p)$. If we apply (2.1) to the binomial expansion

$$
z^{k}=\sum_{m=0}^{k}(-1)^{m}\left(\begin{array}{l}
k \\
m
\end{array}\right)(z+\alpha)^{k-m} \alpha^{m},
$$

and then put $z+\alpha=-\zeta$, we get

$$
\begin{aligned}
\theta^{n}\left(z^{k}\right) & =\sum_{m=0}^{k-1}(-1)^{m}\left(\begin{array}{l}
k \\
m
\end{array}\right) C_{k-m, n}^{(p)}(z+\alpha)^{k-m+n p} \alpha^{m} \\
& =(z+\alpha)^{1+n p} \sum_{m=0}^{k-1}(-1)^{m}\left(\begin{array}{c}
k \\
m
\end{array}\right) \alpha^{m} C_{k-m, n}^{(p)}(-1)^{k-m-1} \zeta^{k-m-1} \\
& =(z+\alpha)^{1+n p}(-1)^{k-1}\left\{\sum_{m=0}^{k-1}\left(\begin{array}{l}
k \\
m
\end{array}\right) \alpha^{m} C_{k-m, n}^{(p)} \zeta^{k-m-1}\right\} \\
& =(z+\alpha)^{1+n p}(-1)^{k-1} P_{k-1}^{(n)}(\zeta), \quad n>1, k \geqslant 1,
\end{aligned}
$$

with $P_{k-1}^{(n)}(\zeta)$ defined in the indicated way. Of course, $P_{k-1}^{(n)}(\zeta)$ also depends on $\alpha$ and $p$, but we suppress this dependence to simplify notation. From the definition of $C_{m n}^{(p)}$ we see that

$$
0 \leqslant C_{m n}^{(p)}<m^{n}[1+(n-1) p]^{n},
$$

and so

$$
\begin{aligned}
\left|P_{k-1}^{(n)}(\zeta)\right| & \leqslant[1+(n-1) p]^{n} \sum_{m=0}^{k}\left(\begin{array}{l}
k \\
m
\end{array}\right)|\alpha|^{m}(k-m)^{n}|\zeta|^{k-m-1} \\
& \leqslant|\zeta|^{-1}[1+(n-1) p]^{n} k^{n}(|\alpha|+|\zeta|)^{k}, \quad n>1, k>1 .
\end{aligned}
$$


For each fixed $\zeta$, then, the power series

$$
G_{n}(\zeta, t)=\sum_{k=1}^{\infty} P_{k-1}^{(n)}(\zeta) t^{k-1}
$$

(which also depends on $\alpha$ and $p$ ) converges at least in the disc $|t|<(|\alpha|+|\zeta|)^{-1}$. Now substitute the defining expression for $P_{k-1}^{(n)}(\zeta)$ into (2.4) and formally interchange the order of summation. This leads to

$$
\begin{aligned}
& \sum_{k=1}^{\infty} P_{k-1}^{(n)}(\zeta) t^{k-1}=\sum_{k=1}^{\infty}\left\{\sum_{r=0}^{k-1}\left(\begin{array}{l}
k \\
r
\end{array}\right) \alpha^{r} C_{k-r, n}^{(p)} \zeta^{k-r-1}\right\} t^{k-1} \\
& =\sum_{k=1}^{\infty} t^{k-1} \sum_{m=1}^{k}\left(\begin{array}{c}
k \\
k-m
\end{array}\right) \alpha^{k-m} C_{m m}^{(p)} \zeta^{m-1} \\
& =\sum_{m=1}^{\infty} C_{m n}^{(p) \zeta^{m-1} t^{m-1}} \sum_{k=m}^{\infty}\left(\begin{array}{c}
k \\
k-m
\end{array}\right) \alpha^{k-m_{t} k-m} \\
& =\sum_{m=1}^{\infty} C_{m n}^{(p)}(\zeta t)^{m-1} \sum_{r=0}^{\infty}\left(\begin{array}{c}
m+r \\
r
\end{array}\right)(\alpha t)^{r} \\
& =\sum_{m=1}^{\infty} C_{m n}^{(p)}(\zeta t)^{m-1}(1-\alpha t)^{-(m+1)} \\
& =(1-\alpha t)^{-2} \sum_{m=1}^{\infty} C_{m n}^{(p)}[(\zeta t) /(1-\alpha t)]^{m-1} .
\end{aligned}
$$

In view of (2.3) it follows that the interchange in order of summation will be justified when $|\alpha t|+|\zeta t|<1$. Note that $|\alpha t|+|\zeta t|<1$ implies $|\zeta t|<1-|\alpha t|$, which implies

$$
\left|\frac{\zeta t}{1-\alpha t}\right|<\frac{1-|\alpha t|}{|1-\alpha t|}<1
$$

In particular, (2.4) and (2.5) are both valid, and we have

$$
\begin{array}{r}
G_{n}(\zeta, t)=\sum_{k=1}^{\infty} P_{k-1}^{(n)}(\zeta) t^{k-1}=(1-\alpha t)^{-2} \sum_{m=1}^{\infty} C_{m n}^{(p)}\left[\frac{\zeta t}{1-\alpha t}\right]^{m-1}, \\
\text { for }|t|<1 /(|\alpha|+|\zeta|) .
\end{array}
$$

Let $\zeta$ be fixed and let the real number $r$ satisfy $0<r<(|\alpha|+|\zeta|)^{-1}$. Then the Cauchy Integral Formula applied to (2.6) gives

$$
P_{k-1}^{(n)}(\zeta)=\frac{1}{2 \pi i} \int_{|t|=r} \frac{G_{n}(\zeta, t)}{t^{k}} d t, \quad n>1, k>1
$$

THEOREM 2.1. Let $f(z)=\sum_{k=0}^{\infty} a_{k} z^{k}$ be analytic in the disc $|z|<R$, where $R>1$, let $r$ satisfy $1<r^{-1}<R$, and let $\zeta$ and $\alpha$ satisfy $|\zeta|+|\alpha|<r^{-1}$. If $z+\alpha=-\zeta$, then

$$
\left(\theta^{n} f\right)(z)=-\frac{(z+\alpha)}{2 \pi i}^{1+n p} \int_{|t|=r} f\left(-\frac{1}{t}\right) G_{n}(\zeta, t) d t, \quad n=1,2,3, \ldots
$$


Proof. Using (2.2) and (2.7), apply $\theta^{n}$ termwise to the power series for $f(z)$ to obtain

$$
\begin{aligned}
\left(\theta^{n} f\right)(z) & =\sum_{k=1}^{\infty} a_{k} \theta^{n}\left(z^{k}\right)=(z+\alpha)^{1+n p} \sum_{k=1}^{\infty}(-1)^{k-1} a_{k} P_{k-1}^{(n)}(\zeta) \\
& =(z+\alpha)^{1+n p} \sum_{k=1}^{\infty} \frac{(-1)^{k-1} a_{k}}{2 \pi i} \int_{|t|=r} \frac{G_{n}(\zeta, t)}{t^{k}} d t .
\end{aligned}
$$

Since $\left|t^{-1}\right|=r^{-1}<R$ in the range of integration, uniform convergence gives

$$
\begin{aligned}
\left(\theta^{n} f\right)(z) & =\frac{(z+\alpha)^{1+n p}}{2 \pi i} \int_{|t|=r}\left\{\sum_{k=1}^{\infty} \frac{(-1)^{k-1} a_{k}}{t^{k}}\right\} G_{n}(\zeta, t) d t \\
& =\frac{(z+\alpha)^{1+n p}}{2 \pi i} \int_{|t|=r}\left\{f(0)-f\left(-\frac{1}{t}\right)\right\} G_{n}(\zeta, t) d t .
\end{aligned}
$$

Since $G_{n}(\zeta, t)$ is analytic for $|t|<(|\alpha|+|\zeta|)^{-1}$, the term involving $f(0)$ drops out, giving (2.8).

The representation (2.8) extends the analogous formula of Hille [6] mentioned earlier in connection with the operator $z d / d z$. The terms in (2.8) are also defined for $p=-1$, and when $\alpha=0$ the equation reduces to the Cauchy Integral Formula for derivatives. However, all the results given below require $p>0$, and so we retain this assumption throughout.

We are now going to replace $\zeta$ in (2.8) by an indexed variable $\zeta_{n}$ so as to make the sequence $G_{n}\left(\zeta_{n}, t\right)$ converge, as $n \rightarrow \infty$. The choice of $\zeta_{n}$ is suggested by the following lemma.

LEMMA 2.1. For fixed $m>1$, and $p>0$, the sequence

$$
S_{m n}^{(p)}=\frac{C_{m n}^{(p)}}{C_{1 n}^{(p)}}\left[\frac{C_{1 n}^{(p)}}{C_{2 n}^{(p)}}\right]^{m-1} \quad(n=1,2,3, \ldots)
$$

is convergent. Moreover $\left(C_{1 n}^{(p)} / C_{2 n}^{(p)}\right) \rightarrow 0, n \rightarrow \infty$.

Proof. If $n=1$, we have

$$
S_{m}^{(p)}=\left(m / 2^{m-1}\right)<1, \quad m>1 .
$$

Next, observe that $S_{m, n+1}^{(p)}$ is obtained from $S_{m n}^{(p)}$ by multiplying by the factor

$$
\frac{m+n p}{1+n p}\left[\frac{1+n p}{2+n p}\right]^{m-1}
$$

We claim that this factor is at most 1 , for that would be equivalent to

$$
1+\frac{m-1}{1+n p}<\left[1+\frac{1}{1+n p}\right]^{m-1},
$$

which is true owing to the binomial theorem. So the terms $S_{m n}^{(p)}$ satisfy $0<S_{m n}^{(p)}<1$ and are monotone decreasing as $n \rightarrow \infty$. The first conclusion follows. As for the second, note that

$$
\frac{C_{2 n}^{(p)}}{C_{1 n}^{(p)}}=\prod_{k=1}^{n}\left[1+\frac{1}{1+(k-1) p}\right]
$$


and this is seen to diverge to $\infty$ by elementary infinite product analysis. This completes the proof.

Define the auxiliary generating functions $H_{n}(x, t)$ by

$$
H_{n}(x, t)=\sum_{m=1}^{\infty} \frac{C_{m n}^{(p)}}{C_{1 n}^{(p)}}\left[\frac{C_{1 n}^{(p)} x t}{C_{2 n}^{(p)}(1-\alpha t)}\right]^{m-1}=\sum_{m=1}^{\infty} S_{m n}^{(p)}\left[\frac{(x t)}{(1-\alpha t)}\right]^{m-1} .
$$

Taking note of (2.6), it is clear that

$$
\begin{aligned}
& H_{n}(x, t)=\frac{(1-\alpha t)^{2}}{C_{1 n}^{(p)}} G_{n}\left(\frac{C_{1 n}^{(p)} x}{C_{2 n}^{(p)}}, t\right) \\
& \text { for }|t|<\frac{1}{|\alpha|+\frac{C_{1 n}^{(p)}}{C_{2 n}^{(p)}}|x|} \text {, or for }|x|<\frac{C_{2 n}^{(p)}}{C_{1 n}^{(p)}}\left(\frac{1}{|t|}-|\alpha|\right) .
\end{aligned}
$$

Then $H_{n}(x, t)$ is analytic in each variable separately in the regions indicated by (2.10).

Recalling that the sequence $S_{m n}^{(p)}$ decreases monotonically with $n$ to some nonnegative limit $S_{m}^{(p)}, 0<S_{m}^{(p)}<S_{m n}^{(p)}<1$, let us define

$$
H(x, t)=\sum_{m=1}^{\infty} S_{m}^{(p)}\left[\frac{(x t)}{(1-\alpha t)}\right]^{m-1} .
$$

Because of its coefficients, (2.11) converges absolutely whenever (2.9) does. For each $n,(2.9)$ converges when the variables satisfy (2.10). Since $H(x, t)$ does not depend on $n$ and $\left(C_{1 n}^{(p)} / C_{2 n}^{(p)}\right) \rightarrow 0, n \rightarrow \infty$, it follows that (2.11) converges for arbitrary $x$ when $t$ is fixed and $|t|<|\alpha|^{-1}$, and for $|t|<|\alpha|^{-1}$ when $x$ is any fixed number. Therefore, $H(x, t)$ is entire in $x$ and analytic in $t$ for $|t|<|\alpha|^{-1}$. Recall that $|\alpha|<1$. Note that we have

$$
H(x, t)=\lim _{n \rightarrow \infty} H_{n}(x, t),
$$

where the convergence is uniform on compact subsets of the admissible regions. Since $S_{1 n}^{(p)}=S_{2 n}^{(p)}=1, n>1$, then

$$
H(x, t)=1+\frac{x t}{1-\alpha t}+\ldots,
$$

so, in particular, $H(x, t) \neq 0$.

Let $f, R$ and $r$ be as in Theorem 2.1. Define

$$
I_{n}(x)=\frac{1}{2 \pi i} \int_{|t|=r} \frac{f\left(-\frac{1}{t}\right) H_{n}(x, t)}{(1-\alpha t)^{2}} d t
$$

where $x$ is such that $(2.10)$ holds with $|t|=r$. That is, (2.13) is defined, and $I_{n}(x)$ is analytic for

$$
|x|<\frac{C_{2 n}^{(p)}}{C_{1 n}^{(p)}}\left(\frac{1}{r}-|\alpha|\right)
$$


Similarly, let

$$
I(x)=\frac{1}{2 \pi i} \int_{|t|=r} \frac{f\left(-\frac{1}{t}\right) H(x, t)}{(1-\alpha t)^{2}} d t .
$$

Then $I(x)$ is entire and, by (2.12), $I_{n}(x) \rightarrow I(x)$ uniformly on bounded sets in the plane. Since $H_{n}(0, t)=H(0, t)=1$, we compute that

$$
I_{n}(0)=I(0)=\frac{1}{2 \pi i} \int_{|t|=r} \frac{f\left(-\frac{1}{t}\right)}{(1-\alpha t)^{2}} d t=-f^{\prime}(-\alpha) .
$$

More generally, the derivatives of $I(x)$ at $x=0$ are given by

$$
\begin{aligned}
I^{(k)}(0) & =\frac{S_{k+1 k !}^{(p)}}{2 \pi i} \int_{|t|=r} \frac{f\left(-\frac{1}{t}\right)}{(1-\alpha t)^{2}}\left(\frac{t}{1-\alpha t}\right)^{k} d t \\
& =-S_{k+1}^{(p)} f^{(k+1)}(-\alpha), \quad k=0,1,2, \ldots
\end{aligned}
$$

A similar result holds for $I_{n}^{(k)}(0)$, with $S_{k+1}^{(p)}$ replaced by $S_{k+1, n}^{(p)}$. Therefore, neither $I_{n}(x)$ nor $I(x)$ vanishes identically unless $f$ is constant. For nonconstant $f$, we can find an integer $u=u(f)$ such that

$$
I_{n}(x)=x^{u} J_{n}(x), \quad I(x)=x^{u} J(x),
$$

where $J_{n}(0) \neq 0$ and $J(0) \neq 0$. Also, there will exist a constant $\gamma_{f}>0$ such that $J(x) \neq 0$ for $|x|<\gamma_{f}$.

TheOREM 2.2. Let $f, R$ and $r$ satisfy the hypothesis of Theorem 2.1, with $f$ nonconstant, and let $0<\gamma<\gamma_{f}$. Then for all $n$ sufficiently large $\left(\theta^{n} f\right)(z)$ has no zero in the disc $|z+\alpha|<\gamma C_{1 n}^{(p)} / C_{2 n}^{(p)}$.

Proof. On the contrary, suppose we could find a subsequence $z_{n_{k}}$ such that $\left(\theta^{n_{k}} f\right)\left(z_{n_{k}}\right)=0$ and $z_{n_{k}}+\alpha=-\zeta_{n_{k}}=-C_{1 n_{k}}^{(p)} x_{n_{k}} / C_{2 n_{k}}^{(p)}$, where $\left|x_{n_{k}}\right|<\gamma$, and where $n_{k}$ is large enough that (2.14) holds for all $k$. Combining (2.8), (2.10), (2.13) and (2.15), there follows

$$
0=\left(\theta^{n_{k}} f\right)\left(z_{n_{k}}\right)=-C_{1 n_{k}}^{(p)}\left(z_{n_{k}}+\alpha\right)^{1+n_{k} p} x_{n_{k}}^{u} J_{n_{k}}\left(x_{n_{k}}\right),
$$

and so $J_{n_{k}}\left(x_{n_{k}}\right)=0$ for all $k$. Since $\left|x_{n_{k}}\right|<\gamma$, yet another subsequence of $\left\{x_{n_{k}}\right\}$ converges to a point $x_{0}$ such that $\left|x_{0}\right|<\gamma<\gamma_{f}$ and $J\left(x_{0}\right)=0$. This contradiction proves the theorem.

REMARK. It may be that $J(x)$ has no zeros, in which case $\gamma_{f}=\infty$. In this situation the discs $0<|z+\alpha|<\gamma C_{1 n}^{(p)} / C_{2 n}^{(p)}$, for every $\gamma>0$, are free of zeros of $\left(\theta^{n} f\right)(z)$ for all $n$ sufficiently large, depending on $\gamma$. Alternatively, $J(x)$ has zeros. If $J\left(x_{0}\right)=0$, we determine by Hurwitz's Theorem a sequence of points $x_{n} \rightarrow x_{0}$ such that $J_{n}\left(x_{n}\right)=0$. If $z_{n}=-\alpha-x_{n}\left(C_{1 n}^{(p)} / C_{2 n}^{(p)}\right)$, then $\left(\theta^{n} f\right)\left(z_{n}\right)=0$ by (2.16), and we also have the asymptotic relation

$$
\frac{C_{2 n}^{(p)}}{C_{1 n}^{(p)}}\left(z_{n}+\alpha\right) \sim\left|x_{0}\right| e^{i\left(\pi+\arg \left(x_{0}\right)\right)}, \quad n \rightarrow \infty .
$$


This is analogous to Theorem 35 of [7, pp. 172-173].

With regard to the asymptotic form of

$$
C_{2 n}^{(p)} / C_{1 n}^{(p)}=\prod_{k=1}^{n}\left[1+\frac{1}{1+(k-1) p}\right]
$$

a straightforward analysis shows that

$$
e(1+n p)^{1 / p} \geqslant C_{2 n}^{(p)} / C_{1 n}^{(p)}>\left(\frac{2+p}{1+p}\right)^{\log (1+n p)^{1 / p}}, \quad p>0 .
$$

As regards neighborhoods of points $z \neq \alpha$, we cannot say as much. Let $\beta$ satisfy $|\beta|<R, \beta \neq-\alpha$, and let $w=T(z)=-\left[p \gamma(z+\alpha)^{p}\right]^{-1}$, where the branch is chosen so as to be analytic at $\beta$. The map is locally invertible, so there exists a function $F(w)$ analytic at $T(\beta)$ such that $f(z)=F(T(z))=F(w)$. By definition of $T(z)$,

$$
\left(\theta^{n} f\right)(z)=\left(D^{n} F\right)(w), \quad n=0,1,2, \ldots,
$$

where $D$ stands for ordinary differentiation. Apply the Whittaker-Gončarov theory to $F(w)$ and translate the information over to the iterates $\left(\theta^{n} f\right)(z)$. Unless $f$ is a polynomial in $(z+\alpha)^{-p}$, there exists a sequence of discs $D_{n}$, shrinking with increasing $n$ to $z=\beta$, and a subsequence $\left\{n_{k}\right\}$ such that $\left(\theta^{n_{k}} f\right)(z)$ has no zero in punctured discs $D_{n_{k}}, k=1,2,3, \ldots$

3. Applications. We consider zeros of successive derivatives of two classes of analytic functions, which correspond to taking $p=1$ and $p=0$ in Theorem 2.2.

Case I: $p=1$. Let $F(w)$ be a function of the type considered by Boas [1] and Widder [7], that is,

$$
F(w)=b_{1} w^{-1}+b_{2} w^{-2}+\ldots, \quad \text { (nonconstant) }
$$

analytic for $|w|>R^{-1}, R>1$. Let $f(z)=F(-1 / z)$, and $(\theta f)(z)=z^{2} f^{\prime}(z)$, so that $\left(\theta^{n} f\right)(z)=\left(D^{n} F\right)(w), n=0,1,2, \ldots$ By Theorem 2.2, the regions $0<|z|<$ $\gamma(n+1)^{-1}$ are eventually zero-free for $\gamma<\gamma_{f}$. Thus for $\gamma<\gamma_{f}$ and all $n$ large, $F^{(n)}(w)$ has no zero which satisfies $\infty>|w|>\gamma^{-1}(n+1)$, and this is the conclusion of Theorem A. Note that $S_{m}^{(1)}=1 /(m-1)$ !, and so $H(x, t)=\exp (x t)$ and

$$
I(x)=\frac{1}{2 \pi i} \int_{|t|=r} F(t) e^{x t} d t .
$$

That is, $I(x)$ is the inverse Laplace transform of $F$. Interpreting (2.17), any zero $x_{0} \neq 0$ of $I(x)$ gives rise to a sequence $\left\{w_{n}\right\}$ of zeros of $F^{(n)}(w)$ which asymptotically approach rays (see [7, Theorem 35])

$$
w_{n} \sim \frac{(n+1) e^{-i \arg \left(x_{0}\right)}}{\left|x_{0}\right|}, \quad n \rightarrow \infty .
$$

Case II: $p=0$. Let $F(w)$ be a function of the form $F(w)=f\left(e^{w}\right)$, where $f(z)$ is analytic in $|z|<R, R>1$. Then $f(z)=F(\ln z)$, and $F(w)$ is analytic in the half-plane $\operatorname{Re}(w)<\ln R$, periodic in the imaginary direction, and tends uniformly to a limit as $\operatorname{Re}(w) \rightarrow-\infty$. Define $\theta$ by $\theta=z d / d z$. Then with $f(z)=F(\ln z)$, we have $\left(\theta^{n} f\right)(z)=\left(D^{n} f\right)(w)$. Theorem 2.2 asserts that constants $\gamma>0$ exist for which the discs $0<|z|<\gamma 2^{-n}$ contain no zeros of $\left(\theta^{n} f\right)(z)$ for all $n$ sufficiently large. 
Equivalently, the region $\operatorname{Re}(w)<\ln \gamma-n \ln 2$ is free of zeros of $F^{(n)}(w)$. To the zeros of $J(x)$ correspond horizontal lines, instead of rays from the origin. If $J\left(x_{0}\right)=0$, then there exists a sequence $\left\{w_{n}\right\}$ such that $F^{(n)}\left(w_{n}\right)=0$ and

$$
w_{n} \sim \ln \left|x_{0}\right|-n \ln 2+i\left(\pi+\arg \left(x_{0}\right)\right), \quad n \rightarrow \infty .
$$

\section{REFERENCES}

1. R. P. Boas, Zeros of successive derivatives of a function analytic at infinity, Univ. Beograd. Publ. Elektrotehn. Fak. Ser. Mat. Fiz. (to appear).

2. J. D. Buckholtz, Successive derivatives of analytic functions, Indiana J. Math. 13 (1971), 83-88.

3. J. D. Buckholtz and J. L. Frank, Whittaker constants, Proc. London Math. Soc. 23 (1971), 348-370.

4. W_, Whittaker constants. II, J. Approximation Theory 10 (2) (1974), 112-122.

5. J. L. Frank and J. K. Shaw, Abel-Goncaroo polynomial expansions, J. Approximation Theory 10 (1) (1974), 6-22.

6. E. Hille, Analytic function theory, Ginn, Boston, Mass., 1962.

7. D. V. Widder, The inversion of the Laplace integral and the related moment problem, Trans. Amer. Math. Soc. 36 (1934), 107-200.

Department of Mathematics, Virginia Polytechic Institute and State University, BlactsBURG, VIRGNA 24061 